\title{
How to be on your guard
} against scams

\section{Sarah Cook}

Head of NHS, Business and General Practice, British Dental Association

cams and phishing schemes are rife in the modern digital age that we live in and are just as likely to affect dental practices as any other business. In addition, criminals have exploited the current pandemic as fraud has increasingly moved online. So, what can you do to protect your business and patients?

The first thing to do is to accept that you can never be completely safe from attack, the best you can hope to do is to minimise your risk.

Next you must access exactly what is at risk? This will be your data and your IT equipment. A dental practice will hold a whole host of data: patient records, staff records, payroll details and so on. In terms of your equipment, your phone line, your computers, your printers and your data back up - do you use a cloud-based system? A good way of assessing this is to record all of this information on an information asset register. This should include details of the support and maintenance arrangements in place, what security measures are in place for each, the quantity of data and how critical it is to the organisation and should be reviewed annually. This will mean that if you were subject to a cyber attack you would for example be able to quickly identify and contact your software supplier to be able to recover the records.

Educating staff and associates on security is also vital. All members of the team must understand the need to keep information secure and be well trained in your policies and procedures. You should keep a list of staff who have access to the various types of information held at the practice (and why) and keep the list updated. You should ensure that staff are suspicious of any emails that ask you to share, change or update any logins or passwords.
In a typical phishing scam, attackers will send fake emails asking for sensitive information or containing a link to a bad website. Some of these phishing emails are difficult to spot. Also remember that phishing and scams come in different forms. You can also get scam text messages, phone calls or social media posts.

A common trick which a dental practice potentially could fall for is for an invoice to be sent for a service that you haven't used. When the attachment is opened, malware is installed on the computer. It is extremely important that staff are cautious, particularly during the current period of uncertainty and additional stress around coronavirus. It is important to take the necessary measures to protect their business and patient data. Remember hackers only need one mistake from one team member for their operation to be a success so everyone must be confident in their ability to spot a scam.

Keep in mind the following tips from NHS Digital:

$\rightarrow$ If you are at all unsure, don’t open any email attachments or click on any links without first ensuring that they are genuine

$\rightarrow$ Check who the email is from, do you know the sender? Look at the email address and not just the sender. If you don't recognise it, be suspicious and try to check through an alternative means of communication and ask them to verify that it's legitimate. For example, is this invoice from a company you have recently used, are you expecting it?

$\rightarrow$ In some phishing schemes, the link you are directed to is different from the one that appears in your email. A way of checking is to hover over the link (without clicking) to see if it looks genuine

$\rightarrow$ If the email is from an official source, it will likely have graphics and images. Do they look legitimate? An official source will never ask you to share personal details or login credentials

$\rightarrow$ Is the email poorly written? Check for spelling and grammatical errors in emails these are often a tell-tale sign of spam.

If you're a NHSmail user and you receive a suspicious email, you can report it using the 'Report Phishing' button on the ribbon within Microsoft Outlook, or forward the email as an attachment, to spamreports@nhs.net.

If you do fall victim to a cyber security attack then you need to know what to do. You should have allocated responsibility for managing data breaches to a dedicated team member, your Data Protection Officer if you are an NHS practice, and all team members should know how to escalate a security incident to the appropriate person. You will need to report certain types of personal data breaches it to the Information Commissioner's Office (ICO) within 72 hours. If you do report a breach, you must explain how it happened, the number of people affected, the likely consequences and how you are dealing with it.

Practice policies and protocols will help you demonstrate compliance with data protection requirements and introduce effective systems in your practice.

$B D A$ members can access further advice about data protection at www.bda.org/gdpr. https://doi.org/10.1038/s41404-020-0550-1 